\title{
Precision RENORM / MBR Diffraction Predictions Tested by Re- cent LHC Results
}

\author{
Konstantin Goulianos ${ }^{1, a}$ \\ ${ }^{1}$ The Rockefeller University, 1230 York Avenue, New York, NY 10065-6399, USA
}

\begin{abstract}
Diffractive, elastic, and precision total pp cross-section predictions, based on the pre-LHC RENORM/MBR model, are compared to recent experimental results. Discrepancies among results by different experiments present a challenge to testing the model. Suggestions for analyses/measurements to understand and resolve the experimental discrepancies are discussed.
\end{abstract}

\section{Introduction}

We present an updated comparison between the precision RENORM / MBR model [1] diffraction predictions and LHC experimantal results, presented at ICNFP-2016, to cover new measurements. Below, we provide a brief historical background of the evolution of the model to a precision tool for data collection and analysis.

In DIS-2015 (Spring 2015) we summarized [2] the pre-LHC predictions of the total, elastic and total-inelastic, as well as the single diffractive (SD) and double diffractive (DD) components of the proton-proton cross section at high energies based RENORM / MBR [1]. We compared the measurements of the SD and DD cross sections from the Tevatron and the LHC with the predictions of the model and found excellent agreement. Good agreement was also observed between the model predictions and the total, elastic and total inelastic cross sections obtained at the Tevatron at $\sqrt{s}=1.8 \mathrm{TeV}$, and at the LHC at $\sqrt{s}=7$ and $8 \mathrm{TeV}$.

The confirmation of the predictions of all the above cross sections at the Tevatron and the LHC up to $\sqrt{s}=8 \mathrm{TeV}$ prompted a calculation/extrapolation to $\sqrt{s}=13 \mathrm{TeV}$, the nominal foreseen collidingbeam energy at the LHC for Summer 2015. For $\sigma_{\text {tot }}, \sigma_{\text {el }}$ and $\sigma_{\text {inel }}$, we predicted $108 \mathrm{mb}, 32 \mathrm{mb}$, and $77 \mathrm{mb}$, respectively, with uncertainties of $\sim 11 \%$ in all cases, mainly due to the uncertainty in the energy-squared scale parameter $s_{0}$ of the model.

In Summer 2015 we updated the energy-squared scale parameter $s_{0}$ of RENORM / MBR to a more precise value based on a tensor glueball interpretation of the Axial Field Spectrometer (AFS) exclusive charged di-pion data [3-5]. This change in RENORM / MBR decreases the uncertainties in the predictions of the total, elastic, and total-inelastic cross sections to less than $2 \%$ from Tevatron to LHC energies, with little or no effect on the mean values. The predictions were compared with measurements by ATLAS at $\sqrt{s}=7 \mathrm{TeV}$ and by TOTEM at $\sqrt{s}=7$ and $8 \mathrm{TeV}$ at Moriond QCD in March 2016 [6] and found to be in good agreement.

\footnotetext{
a e-mail: dino@rockefeller.edu
} 
At ICNFP-2016 (July 2016) we presented an update of [6] to include the new measurements of the total inelastic cross section at $\sqrt{s}=13 \mathrm{TeV}$ by ATLAS and CMS reported at DIS-2016 (April 2016) [7]. All measured cross sections were in good agreement within the experimental uncertainties of the data and the theoretical uncertainties of the model, reaching down to the $\sim 1 \%$ level (Table 1). However, there were some disagreements among the measurements themselves and with the RENORM/MBR predictions at the $\sim 2 \sigma$ level.

We recalled that in RENORM the total inelastic cross section is calculated as the difference between the total and the elastic cross sections, and thus, a measured lower $\sigma_{\mathrm{el}}$ would result in a higher $\sigma_{\text {inel }}$. As can be seen in Table 1, at ICNFP-2016 the MBR $\sigma_{\text {el }}$ was larger than the TOTEM and CMS measurements by $\sim 2 \mathrm{mb}$ at $\sqrt{s}=7 \mathrm{TeV}$, which would imply a higher MBR prediction for $\sigma_{\mathrm{el}}$ at $13 \mathrm{TeV}$ by $\sim 2 \mathrm{mb}$ as well. We recommended that the interplay between $\sigma_{\mathrm{el}}$ and $\sigma_{\text {inel }}$ be carefully taken into consideration as more measurements of both $\sigma_{\mathrm{el}}$ and $\sigma_{\text {tot }}$ at $\sqrt{s}=8 \mathrm{TeV}$ and $\sqrt{s}=13 \mathrm{TeV}$ with reduced luminosity and extrapolation uncertainties became available.

After INNFP-2016, preliminary new results were presented at ICHEP-2016 (August) [8] (cf. Table 1). Notable among them are the updated measurements at $\sqrt{s}=13 \mathrm{TeV}$ by ATLAS of $\sigma_{\text {inel }}^{\text {ATLAS }}=79.3 \pm 0.6$ (exp) \pm 2.5 (extr) \pm 1.3 (lum) and CMS $\sigma_{\text {inel }}^{\text {CMS }}=71.3 \pm 0.5$ (exp) \pm 2.7 (extr) \pm 2.1 (lum). The new results are compatible at the $2 \sigma$ level of all the uncertainties combined in quadratute.

A new measurement by TOTEM of $\sigma_{\text {tot }}, \sigma_{\text {el }}$ and $\sigma_{\text {inel }}$ at $\sqrt{s}=2.76 \mathrm{TeV}$ was presented at DIS2017 (April 2017) [9] (cf. Table 1). The data are in excellent agreement with the RENORM / MBR predictions down to the $\sim 1 \%$ level.

\section{RENORM cross sections}

The total, elastic, and total-inelastic cross sections in the RENORM / MBR model depend on the value of the energy-squared scale parameter, $s_{0}$. Quoting verbatim from Ref. [2],

"The total cross section $\left(\sigma_{\text {tot }}\right)$ is expressed as [10]

$$
\begin{array}{lrl}
\sigma_{\text {tot }}^{p^{ \pm} p}= & 16.79 s^{0.104}+60.81 s^{-0.32} \mp 31.68 s^{-0.54} & \text { for } \sqrt{s} \leq 1.8 \mathrm{TeV}, \\
\sigma_{\text {tot }}^{p^{ \pm} p}= & \sigma_{\text {tot }}^{\mathrm{CDF}}+\frac{\pi}{s_{0}}\left[\left(\ln \frac{s}{s_{F}}\right)^{2}-\left(\ln \frac{s^{\mathrm{CDF}}}{s_{F}}\right)^{2}\right] & \text { for } \sqrt{s} \geq 1.8 \mathrm{TeV},
\end{array}
$$

where $s_{0}$ and $s_{F}$ are the energy and (Pomeron flux) saturation scales, $s_{0}=3.7 \pm 1.5 \mathrm{GeV}^{2}$ and $\sqrt{s}_{F}=22 \mathrm{GeV}$, respectively. For $\sqrt{s} \leq 1.8 \mathrm{TeV}$, where there are Reggeon contributions, we use the global fit expression [11], while for $\sqrt{s} \geq 1.8 \mathrm{TeV}$, where Reggeon contributions are negligible, we employ the Froissart-Martin formula [12-14]. The two expressions are smoothly matched at $\sqrt{s} \approx 1.8$ $\mathrm{TeV}$. The $\sigma_{\mathrm{el}}$ for $\sqrt{s} \leq 1.8 \mathrm{TeV}$ is obtained from the global fit [11], while for $1.8<\sqrt{s} \leq 50 \mathrm{TeV}$ we use an extrapolation of the global-fit ratio of $\sigma_{\text {el }} / \sigma_{\text {tot }}$, which is slowly varying with $\sqrt{s}$, multiplied by $\sigma_{t o t}$. The total non-diffractive cross section is given by $\sigma_{\mathrm{ND}}=\left(\sigma_{\mathrm{tot}}-\sigma_{\mathrm{el}}\right)-\left(2 \sigma_{\mathrm{SD}}+\sigma_{\mathrm{DD}}+\sigma_{\mathrm{CD}}\right)$."

\subsection{Tensor glueball exchange predictions vs. measurements}

The partial wave analysis of the AFS exclusive $\pi^{ \pm}$data [5], performed in terms of a fit with a model with S-wave and D-wave amplitudes as a function of the di-pion mass up to $2.3 \mathrm{GeV}$, leads to the results presented in Figure 1.

The D-wave dominates at masses above $\sim 2 \mathrm{GeV}$, and according to the presumed interpretation in Ref. [5] it corresponds to a spin-2 tensor glueball of mass $M_{\mathrm{tgb}}$. A Gaussian fit to this enhancement yields $\mathrm{M}_{\mathrm{tgb}}=2.10 \pm 0.68 \mathrm{GeV}$. Identifying $\mathrm{M}_{\mathrm{tgb}}^{2}$ with the saturated glueball-like enhancement of the MBR parameter $s_{0}$ (see Eq. 2) yields $s_{0}=4.42 \pm 0.34 \mathrm{GeV}^{2}$. Using this value in Eq. 2 we predicted 


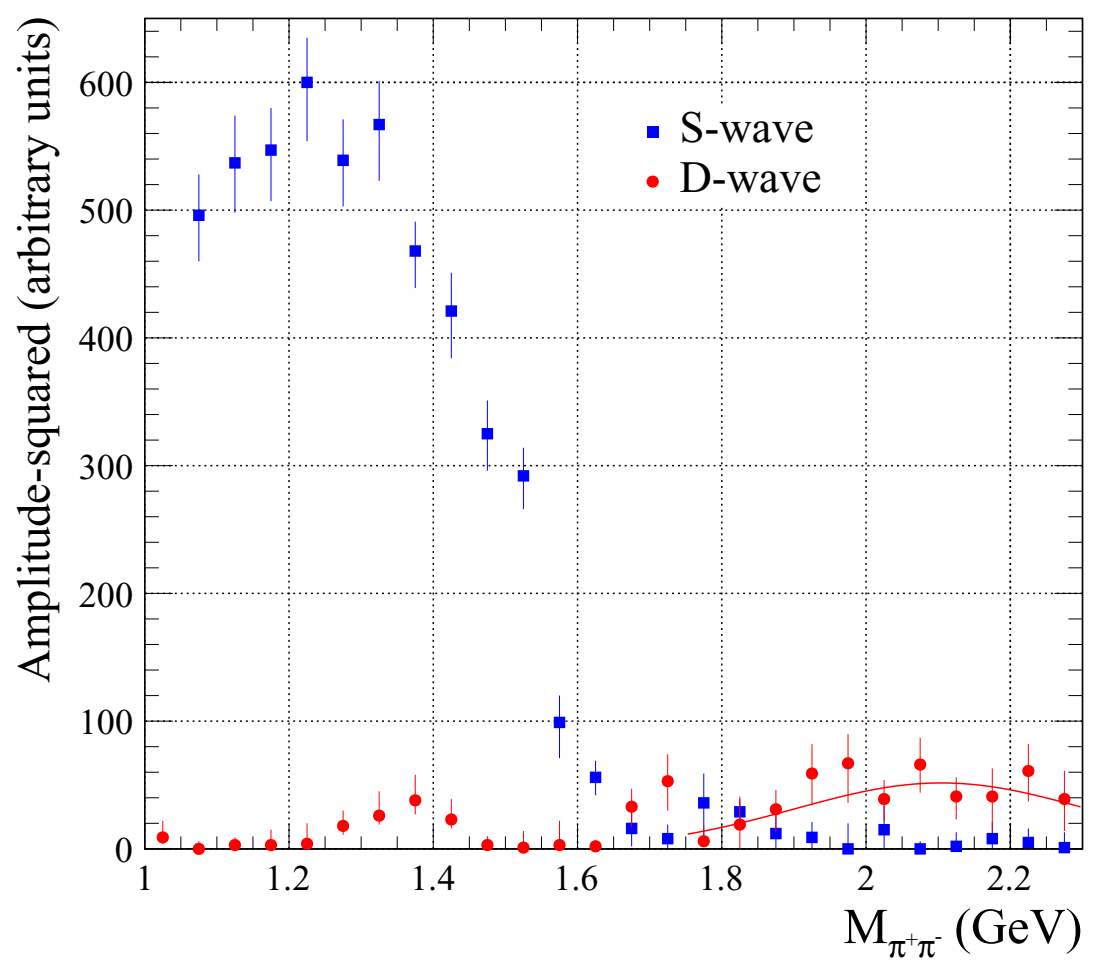

Figure 1. Extraction of tensor-glueball-exchange parameters from a Gaussian fit to the exclusive $\pi^{ \pm}$Axial Field Spectrometer data. The fit yields a mean mass value $\left\langle M_{\pi^{+} \pi^{-}}\right\rangle=2.10 \mathrm{GeV}$ of width $\Delta= \pm 0.68 \mathrm{GeV}$.

for $\sigma_{\text {tot }}, \sigma_{\text {el }}$, and $\sigma_{\text {inel }}$ at $13 \mathrm{TeV}$ cross sections of $103.7 \pm 1.9 \mathrm{mb}, 30.2 \pm 0.8 \mathrm{mb}$, and $73.5 \pm 1.3 \mathrm{mb}$, respectively. The ATLAS- and TOTEM-measured cross sections at $\sqrt{s}=7$ and $8 \mathrm{TeV}$ [15-17] are shown in Table 1 along wiith the MBR predictions.

The ICNFP-2016, ATLAS and CMS cross-section are imported from Ref. [7]. Details of the ATLAS results, as presented in [7], are listed below:

1. Elastic cross section:

$$
\sigma_{\mathrm{el}}^{\mathrm{ALF}}(7 \mathrm{TeV})=24.00 \pm 0.19(\text { stat }) \pm 0.57 \text { (syst) } \mathrm{mb}
$$

2. Nuclear slope:

$$
b^{\mathrm{ALFA}}(7 \mathrm{TeV})=19.73 \pm 0.14(\text { stat }) \pm 0.26(\text { syst }) \mathrm{GeV}^{-2}
$$

3. Total cross section

$$
\sigma_{\text {tot }}^{\mathrm{ALFA}}(7 \mathrm{TeV}=95.35 \pm 0.38(\text { stat }) \pm 1.25(\exp ) \pm 0.37(\text { extr }) \mathrm{mb}
$$

4. Inelastic cross section:

- $\sigma_{\text {inel }}^{\mathrm{ALFA}}(7 \mathrm{TeV})=71.34 \pm 0.36$ (stat) \pm 0.83 (syst) $\mathrm{mb}$

- $\sigma_{\text {inel }}^{\mathrm{MBTS}}(7 \mathrm{TeV})=69.4 \pm 2.4(\exp ) \pm 6.9$ (extr) $\mathrm{mb}$

- $\sigma_{\text {inel }}^{\mathrm{MBTS}}(13 \mathrm{TeV})=73.1 \pm 0.9(\exp ) \pm 3.8$ (extr) \pm 6.6 (lum) $\mathrm{mb}$ 
Table 1. The total, elastic, and total inelastic MBR predictions (in mb) at $\sqrt{s}=7,8$ and $13 \mathrm{TeV}$ presented at ICNFP-2016 vs. measurements at the LHC by TOTEM and ATLAS. Also shown are updated results by ATLAS for $\sqrt{s}=8$ and $13 \mathrm{TeV}$ and CMS for $\sqrt{s}=13 \mathrm{TeV}$ presented at ICHEP-2016, and TOTEM results for $\sqrt{s}=2.6 \mathrm{TeV}$ presented at DIS-2017.

\begin{tabular}{cllll}
\hline $\begin{array}{c}\sqrt{s} \\
(\mathrm{TeV})\end{array}$ & MBR Experiment & $\begin{array}{l}\sigma_{\text {tot }} \\
(\mathrm{mb})\end{array}$ & $\begin{array}{l}\sigma_{\text {el }} \\
(\mathrm{mb})\end{array}$ & $\begin{array}{l}\sigma_{\text {inel }} \\
(\mathrm{mb})\end{array}$ \\
\hline 7 & MBR & $95.4 \pm 1.2$ & $26.4 \pm 0.3$ & $69.0 \pm 1.0$ \\
& TOTEM & $98.3 \pm 2.9$ & $24.8 \pm 1.3$ & $73.7 \pm 1.7$ \\
& TOTEM_Lum_ind & $98.0 \pm 2.5$ & $25.2 \pm 1$ & $72.9 \pm 1.5$ \\
& ATLAS & $95.35 \pm 1.36$ & $24.00 \pm 0.60$ & $71.34 \pm 0.90$ \\
\hline 8 & MBR & $97.1 \pm 1.4$ & $27.2 \pm 0.4$ & $69.9 \pm 1.0$ \\
& TOTEM & $101.7 \pm 2.9$ & $27.1 \pm 1.4$ & $74.7 \pm 1.7$ \\
& ATLAS-ICHEP16 & $96.1 \pm 0.9$ & $27.1 \pm 1.4$ & $74.7 \pm 1.7$ \\
\hline 13 & MBR & $103.7 \pm 1.9$ & $30.2 \pm 0.8$ & $73.5 \pm 1.3$ \\
& ATLAS & $\mathrm{xxx}$ & $\mathrm{xxx}$ & $73.1 \pm 0.9(\mathrm{exp}) \pm 3.8($ extr) $\pm 6.6(\mathrm{lum})$ \\
& ATLAS-ICHEP16 & $\mathrm{xxx}$ & $\mathrm{xxx}$ & $79.3 \pm 0.6(\mathrm{exp}) \pm 2.5(\mathrm{extr}) \pm 1.3(\mathrm{lum})$ \\
& CMS-ICHEP16 & $\mathrm{xxx}$ & $\mathrm{xxx}$ & $71.3 \pm 0.5(\mathrm{exp}) \pm 2.7(\mathrm{extr}) \pm 2.1(\mathrm{lum})$ \\
\hline 2.6 & MBR & $85.2 \pm 1.2$ & $21.7 \pm 0.3$ & $63.5 \pm 1.1$ \\
& TOTEM-DIS17 & $84.7 \pm 3.3$ & $21.8 \pm 1.4$ & $62.8 \pm 2.9$ \\
\hline
\end{tabular}

Shown in Table 1 is also a measurement of the total inelastic cross section by ATLAS at $\sqrt{s}=$ $13 \mathrm{TeV}[18], \sigma_{\text {inel }}=73.1 \pm 0.9$ (exp) \pm 3.8 (extr) \pm 6.6 (lum) $\mathrm{mb}$, which, apart from the extrapolation and luminosity uncertainties, is in excellent agreement with MBR. All the other measurements are also in good agreement with the predictions. The tensor-glueball-based prediction of $\sigma_{\text {inel }}$ at $\sqrt{s}=13 \mathrm{TeV}$ agrees with the ATLAS measurement at the $1 \%$ level.

Following ICNFP-2016, new results were presented at ICHEP-2016 [8] by ATLAS and CMS, and at DIS-2017 [7] by TOTEM (cf. Table 1). As discussed in the introduction, these results confirm the predictions of RENORM / MBR.

\section{Summary and conclusions}

We present the predictions of the total, elastic scattering, and total-inelastic proton-proton cross sections at $\sqrt{s}=7,8$ and $13 \mathrm{TeV}$ of the RENORM / MBR MBR model, based on a Regge-theory inspired tensor-glueball implementation of the RENORM model for hadronic diffraction, and compare them with experimental results by the TOTEM, ATLAS, and CMS Collaborations. All measured cross sections are in good agreement within the experimental uncertainties of the data and the theoretical uncertainties of the model, reaching down to the $\sim 1 \%$ level.

\section{Acknowledgments}

Warmly acknowledged are Dr. Michael Albrow for providing a copy of the thesis of Peter C. Cesil, where the tensor-Pomeron data and analysis are discussed, and Dr. Robert Ciesielski for extracting the tensor-Pomeron parameters from the data and for many invaluable discussions. 


\section{References}

[1] K. Goulianos, Diffraction in QCD [arXiv:hep-ph/0203141]

K. Goulianos, Hadronic diffraction: where do we stand? [arXiv:hep-ph/0407035].

[2] Konstantin Goulianos, Review of RENORM Diffractive Predictions for LHC up to $8 \mathrm{TeV}$ and Extension to $13 \mathrm{TeV}$, PoS(DIS 2015)073.

[3] M.G. Albrow, T.D. Coughlin, J.R. Forshaw, Prog. Part. Nucl. Phys. 65, 149 (2010) [DOI:10.1016/j.ppnp.2010.06.001 [arXiv:1006.1289].

[4] M.G. Albrow, private communication, discussions about the Axial Field Spectrometer results on exclusive $\pi^{+} \pi^{-}$.

[5] Peter Charles Cesil, A Search for Centrally Produced Glueballs in Proton-Proton Interactions, $\mathrm{PhD}$ thesis, University of Cambridge, September 1984.

[6] K. Goulianos, RENORM tensor-Pomeron diffractive predictions, in 51st Rencontres de Moriond on QCD and High Energy Interactions, 19-26 Mar 2016. La Thuile, Italy C16-03-19.1 [arXiv:1606.01302v2 [hep-ph] 3 Jun 2016].

[7] Presented at 24th International Workshop on Deep-Inelastic Scattering and Related Subjects (DIS 2016), Hamburg, Germany, April 11-25, 2016 [https://dis2016.desy.de/]:

(a) Maciej Trzebinzki (ATLAS Collaboration), Measurements of Total, Elastic, Inelastic and Diffractive Cross Sections with the ATLAS Detector;

(b) Hans Van Haevermaet (CMS Collaboration), Measurement of the inelastic cross section at $13 \mathrm{TeV}$.

[8] Presented at 38th International Conference on High Energy Physics (ICHEP 2016), Chicago, IL, USA August 03-10 2016 [https://www.ichep2016.org/]. T. Sykora (Charles University in Prague, on behalf of the ATLAS, CMS, LHCb and TOTEM collaborations, Total, elastic and inelastic pp cross sections at the LHC [https://indico.cern.ch/event/432527/contributions/1072410/].

[9] Presented at XXV International Workshop on Deep-Inelastic Scattering and Related Subjects (DIS 2017, University of Birmingham, Birmingham, UK, April 3-7 2017 F. J. Nemes (TOTEM Collaboration), Elastic and Total Cross-Section Measurements by TOTEM: Past and Future, [PoS(DIS2017)059].

[10] R. Ciesielski and K. Goulianos, MBR Monte Carlo Simulation in PYTHIA-8 [arXiv:1205.1446].

[11] R. J. M. Covolan, J. Montanha and K. Goulianos, Phys. Lett. B 389, 176 (1996).

[12] M. Froissart, Phys. Rev. 3, 123 (1961).

[13] A. Martin, Nuovo Cimento 42, 930 (1966).

[14] A. Martin, Phys. Rev. D 80, 065013 (2009).

[15] Marek Tasevsky (ATLAS Collaboration), Diffraction and forward physics of the ATLAS experiment from Run I, Diffraction 2014, AIP Conf. Proc. 1654 (2015) 040002, (2015-04-10) [DOI:10.1063/1.4915967].

[16] G. Antchev et al. (TOTEM Collaboration), Eur. Phys. Lett. 101 (2013) 21002, Eur. Phys. Lett. 101 (2013) 21004, Eur. Phys. Lett. 101 (2013) 21003.

[17] G. Antchev et al. (TOTEM Collaboration), Phys. Rev. Lett. 111, 012001 (2013).

[18] D. Schaefer (CERN), Recent soft QCD results from the LHC, in 2016 Aspen Winter Conference in Particle Physics: Particle Physics on the Verge of Another Discovery?, 11-16 January 2016, ACP, Aspen, CO. 